\title{
Human milk oligosaccharides are differentially metabolised in neonatal rats
}

\author{
Evelyn Jantscher-Krenn, Carolin Marx and Lars Bode* \\ Division of Neonatal Medicine and Division of Pediatric Gastroenterology and Nutrition, Department of Pediatrics, \\ University of California, 200 West Arbor Drive, MC 8450, San Diego, CA 92103-8450, USA
}

(Submitted 26 March 2012 - Final revision received 12 November 2012 - Accepted 26 November 2012 - First published online 14 January 2013)

\begin{abstract}
Human milk oligosaccharides (HMO) are complex glycans that are highly abundant in human milk, but not in infant formula. Accumulating data, mostly from in vitro and animal studies, indicate that HMO benefit the breast-fed infant in multiple ways and in different target organs. In vitro incubation studies suggest that $\mathrm{HMO}$ can resist the low $\mathrm{pH}$ in the infant's stomach and enzymatic degradation in the small intestine and reach the colon in the same composition as in the mother's milk. The oligosaccharide composition in faeces of breast-fed infants is, however, very different from that in the mother's milk, raising questions on when, where and how HMO are metabolised between ingestion and excretion. To answer some of these questions, we established a pulse-chase model in neonatal rats and analysed HMO profiles to track their composition over time in five consecutive equal-length intestinal segments as well as in serum and urine. The relative abundance of individual HMO changed significantly within the first $2 \mathrm{~h}$ after feeding and already in the segments of the small intestine prior to reaching the colon. Only $3^{\prime}$-sialyllactose, the major oligosaccharide in rat milk, and hardly any other HMO appeared in the serum and the urine of HMO-fed rats, indicating a selective absorption of rat milk-specific oligosaccharides. The present results challenge the paradigm that HMO reach the colon and other target organs in the same composition as originally secreted with the mother's milk. The present results also raise questions on whether rats and other animals represent suitable models to study the effects of $\mathrm{HMO}$.
\end{abstract}

Key words: Breast milk: Oligosaccharides: Digestion: Absorption: Excretion

With every feeding, breast-fed infants ingest high amounts of unconjugated, complex glycans called human milk oligosaccharides (HMO). A volume of 1 litre of mature human milk contains between 10 and $20 \mathrm{~g}$ of HMO, and the concentrations in colostrum can be even higher (reviewed in Bode ${ }^{(1,2)}$, Kunz et $a{ }^{(3)}{ }^{(3)}$. A large body of evidence indicates that HMO benefit the breast-fed infant in multiple ways that reach from protection against infections ${ }^{(2-4)}$ and augmentation of gut maturation $^{(5,6)}$ to enhancement of brain development ${ }^{(7)}$ and protection from necrotising enterocolitis ${ }^{(8)}$. Most of the supporting data stem from studies that isolate HMO from human milk and then directly assess their effects in in vitro or ex vivo models. This approach, however, is based on the assumption that HMO reach the target organs and tissues in the same composition as originally excreted with the milk, which may not be the case.

HMO consist of glucose, galactose (Gal), $N$-acetylglucosamine, fucose and sialic acid. All HMO carry lactose (Gal $\beta 1-4$ glucose) at the reducing end and can be elongated with up to fifteen heteromeric $\mathrm{Gal} / \mathrm{N}$-acetylglucosamine disaccharides.
Lactose or the linear or branched oligosaccharides can be further fucosylated and/or sialylated in various different linkages, leading to a diverse pool of complex oligosaccharides $^{(1,3,9)}$. More than 100 different HMO have been characterised so far ${ }^{(9)}$, but not each and every woman produces the entire set of HMO, which depends highly on genetic and, potentially, environmental factors and changes over the course of lactation. At least some of their beneficial effects are highly structure-dependent and are ablated or abolished when HMO are partially degraded or structurally modified ${ }^{(8,10-14)}$.

On the one hand, in vitro studies show that HMO resist a low $\mathrm{pH}$ that resembles conditions in the infant's stomach, and that HMO are only minimally degraded by the pancreatic and brush-border enzymes that model the infant's proximal small intestine $e^{(15,16)}$. On the other hand, in vitro data and most recent in vivo studies in mice strongly suggest that certain bacteria in the infant's intestine are capable of metabolising $\mathrm{HMO}^{(17-20)}$. Comprehensive analysis of infant faeces confirmed partial HMO degradation and bioconversion in the infant's gastrointestinal tract, resulting in profiles remarkably

Abbreviations: 2AB, 2-aminobenzamide; 2'FL, 2'-fucosyllactose; $3^{\prime} \mathrm{SL}, 3^{\prime}$-sialyllactose; DF, dam-fed; FF, formula alone; Gal, galactose; GOS, galacto-oligosaccharides; HMO, human milk oligosaccharides; LNFP1, lacto- $N$-fucopentaose 1.

* Corresponding author: L. Bode, fax +1 619543 7537, email lbode@ucsd.edu 
different from the HMO profiles in their mother's milk ${ }^{(21-23)}$. When and where along the digestive tract HMO are degraded and modified remain unknown.

Active and passive mechanisms facilitate the transport of HMO across monolayers of human intestinal epithelial cells ${ }^{(24)}$. In addition, elegant ${ }^{13} \mathrm{C}$-labelling studies show that HMO appear in the urine of breast-fed infants ${ }^{(25,26)}$. The combined in vitro and in vivo data suggest that approximately $1 \%$ of the ingested HMO are absorbed, reach the systemic circulation and are excreted with the urine. The HMO composition in the urine, however, is not identical to that of the corresponding milk.

Animal models are being used increasingly to investigate the potential biological effects of $\mathrm{HMO}^{(8,14,20,27)}$, but little is known about their kinetics of absorption and metabolism. Here, we used an in vivo pulse-chase digestion model in neonatal rats and monitored the HMO composition over time in five consecutive equal-length intestinal segments between the pylorus and the anus, as well as in the blood and urine. In the same model, we also determined the absorption and metabolism of galacto-oligosaccharides (GOS) that are commonly added to infant formula in an attempt to mimic some of the beneficial effects of HMO. So far, the common notion had been that both HMO and GOS reach the colon intact, where they serve as prebiotics and are metabolised by certain bacteria $^{(3,4,28)}$. Our data suggest that the structural composition of HMO already changes in proximal parts of the intestine and that the structures originally found in human milk or infant formula may in fact not always reach the target organs or tissues as originally anticipated.

\section{Materials and methods}

\section{Human milk oligosaccharide standards and} galacto-oligosaccharides

$2^{\prime}$-Fucosyllactose (2'FL), 3-fucosyllactose, $3^{\prime}$-sialyllactose ( $\left.3^{\prime} \mathrm{SL}\right)$, $6^{\prime}$-sialyllactose, lacto- $N$-tetraose and lacto- $N$-fucopentaose 1 (LNFP1) were purchased from V-Labs, Inc. GOS syrup (Vivinal $^{\circledR}$, DM 75\%) was kindly provided by Friesland Campina Domo. Apart from 59\% GOS, the product also contains $21 \%$ lactose, $19 \%$ glucose and $1 \%$ Gal.

\section{Isolation of human milk oligosaccharides}

Healthy lactating women were recruited at the UCSD Medical Center, San Diego, CA, and human milk was collected after written informed consent was obtained, and the research was approved by the University's Institute Review Board. Oligosaccharides were isolated from the pooled human milk, as previously reported ${ }^{(8,12)}$. Briefly, following delipidation, proteins were precipitated from the aqueous phase by addition of ice-cold ethanol and subsequent centrifugation. Ethanol was evaporated from the HMO-containing supernatant under vacuum. Lactose and salts were removed by size-exclusion chromatography over a BioRad P2 column $(100 \mathrm{~cm} \times 16 \mathrm{~mm}$; Bio-Rad) using a semi-automated fast protein liquid chromatography system.

\section{Animals}

Institutional and national guidelines for the care and use of animals were followed, and all experimental procedures involving animals were approved by the Institutional Animal Care and Use Committee at the University of California, San Diego, CA. Time-pregnant Sprague-Dawley rats were purchased from Harlan. After birth, pups were housed with the dam. On the third day of life, pups were separated from the dam and fasted for $12 \mathrm{~h}$ overnight in a humidified incubator at $34^{\circ} \mathrm{C}$. Control animals stayed with the dam (dam-fed, DF). A group of pups was killed immediately after the fasting period and served as fasted baseline control. Other pups were orally adminstered with $300 \mu \mathrm{l}$ formula alone (FF), formula with $\mathrm{HMO}(\mathrm{FF}+\mathrm{HMO}, 15 \mathrm{mg} / \mathrm{ml})$ or formula with GOS $(\mathrm{FF}+\mathrm{GOS}, 8 \mathrm{mg} / \mathrm{ml})$. Oral administration was performed with a twenty-four gauge L-Cath Peel Away System PICC catheter (Becton Dickinson Infusion Therapy Systems, Inc.) ${ }^{(8,29)}$. A $2 \mathrm{~cm}$ mark on the catheter ensured that all catheters were inserted and placed correctly. Slight resistance prior to reaching the $2 \mathrm{~cm}$ mark indicated that the catheter was wrongfully placed into the trachea instead of the oesophagus. In that case, the catheter was removed and insertion repeated. Once the catheter was placed correctly, $300 \mu$ l formula was slowly injected through an attached syringe. Afterwards, the catheter was removed slowly. Rat formula contained $15 \mathrm{~g}$ Similac 60/40 (Ross Pediatrics) and $75 \mathrm{ml}$ Esbilac (Pet-Ag, Inc.). Pups were euthanised at different times post-feeding ( $n 3$ for each time point). Blood was collected from the decapitation site. Urine was aspirated from the bladder. Entire intestines (from the pyloric opening to the anal opening) were removed. Since it was difficult to consistently and reliably differentiate anatomical sites (duodenum, jejunum, ileum, caecum and colon) at the third day of life, entire intestines were cut in five equal-length sections. Sections $1-3$ represent the small intestine with the duodenum and jejunum. Section 4 represents distal parts of the jejunum and ileum. Section 5 represents mostly the colon. For some experiments, luminal content from the different sections was directly aspirated with a $10 \mu \mathrm{l}$ pipette tip, flash frozen and stored at $-80^{\circ} \mathrm{C}$ ( $n 3$ for each time point). A volume of $0.5 \mu$ l luminal content was sufficient for HPLC analysis, which allowed for sampling without compromising the integrity of the intestinal wall and guaranteed that our luminal content sample was free of intestinal tissue. For most other experiments, entire intestinal tissue sections that included the luminal content were placed in a tube with $500 \mu \mathrm{l}$ water and immediately heated for $5 \mathrm{~min}$ at $100^{\circ} \mathrm{C}$ to inactivate intestinal and intracellular enzymes. After sonication and centrifugation, supernatants were stored at $-80^{\circ} \mathrm{C}$.

\section{Isolation and purification of human milk oligosaccharides} and galacto-oligosaccharides from animal specimens

To isolate pre-labelled HMO or unlabelled HMO, intestinal tissue samples were centrifuged twice at $6800 \mathrm{~g}$ to remove tissue debris and lipids. Intestinal tissue $(500 \mu \mathrm{l})$ or serum ( $50 \mu \mathrm{l}$ diluted in $400 \mu \mathrm{l}$ water) samples were cleaned up 
using the chloroform-methanol method, as previously described for isolation of glycans from milk samples ${ }^{(8,12)}$ with the following modifications. A volume of $1 \mathrm{ml}$ chloroform was added to the samples and centrifuged at $1300 \boldsymbol{g}$ for $15 \mathrm{~min}$. The aqueous phase was transferred into fresh microtubes and centrifuged again after the addition of $800 \mu \mathrm{l}$ chloroform-methanol. Following transfer to a fresh tube, $300 \mu \mathrm{l}$ water were added to the aqueous phase to precipitate more proteins. Samples were centrifuged again and the supernatant was further cleaned up using C18 and carbograph spin columns, as previously described ${ }^{(8,12)}$. Luminal contents were diluted in the ratio 1:4000 with water, and subjected to C18 and carbograph spin columns. Urine samples $(50 \mu \mathrm{l})$ were also cleaned up using C18 and carbograph spin columns without prior chloroform-methanol extraction.

\section{Profiling of human milk oligosaccharides and galacto- oligosaccharides by HPLC with fluorescence detection}

Recovered, dried HMO and GOS from the intestinal samples were fluorescently labelled with 2-aminobenzamide (2AB), as previously described ${ }^{(8,12)}$. The $2 \mathrm{AB}$-glycans were separated by HPLC with fluorescence detection on an amide-80 column ( $4.6 \mathrm{~mm}$ inner diameter $\times 25 \mathrm{~cm}, 5 \mu \mathrm{m}$; Tosoh Bioscience), with a linear gradient of a $50 \mathrm{~mm}$-ammonium formate/ acetonitrile buffer system. Separation was performed at $25^{\circ} \mathrm{C}$ and monitored by a fluorescence detector at $360 \mathrm{~nm}$ excitation and $425 \mathrm{~nm}$ emission. Standard retention times and MS analysis were used to annotate HPLC peaks. The amount of each individual HMO was calculated based on pre-determined response factors. Relative abundance of each of the individual HMO was determined by setting the sum of the thirteen major HMO as $100 \%$ total HMO.

\section{Analysis of human milk oligosaccharides by MS}

HPLC peak-fraction samples were analysed by MS on a Thermo LCQ Duo Ion trap mass spectrometer equipped with a Nano-ESI-source. MS was conducted in positive ion mode and sodiated ions were detected with $\mathrm{m} / z$ values increased by $120 \mathrm{Da}$ corresponding to the $2 \mathrm{AB}$ label. All mass spectra were acquired over approximately $30 \mathrm{~s}$ in a mass/charge range of 300 to $2000 \mathrm{Da}$.

\section{Analysis of human milk oligosaccharides by enzymatic digest}

To confirm the identity of individual peaks in urine, 2AB-labelled samples were either treated with $\alpha 2-3,6,8$, 9-neuraminidase (Sigma) or $\alpha 1$-2-fucosidase according to the manufacturer's instructions (both Sigma). Enzyme-treated and untreated samples were run in parallel and analysed by HPLC with fluorescence detection as described above.

\section{Statistical analysis}

Relative abundance of oligosaccharides in the different intestinal segments and at different time points were calculated as means and standard deviations, with $n 3$ rat pups per time point. Differences in the relative abundance of each oligosaccharide between different intestinal segments at a given time or between different time points in the same segment were calculated by one-way ANOVA (Prism 5 for Mac OS X; GraphPad Software). $P$ values $<0.05$ were considered statistically significant.

\section{Results}

Human milk oligosaccharides can be detected in rat intestines, serum and urine without interference from other dietary or endogenous oligosaccharides and without degradation during sample processing

Prior to determining the fate of HMO during intestinal passage, we established a method to measure HMO composition in different intestinal sections at different time points. The method needed to distinguish HMO from other exogenous dietary oligosaccharides present in rat milk or formula, as well as from endogenous oligosaccharides, e.g. on intestinal epithelial cells. The method also needed to ensure that HMO are not degraded by intracellular glycosidases that may be released during tissue disruption in the sample collection process.

First, we assessed whether HMO can be distinguished from endogenous oligosaccharides and whether HMO are degraded or modified during tissue processing. Fasted rat pups received an HMO feed $(15 \mathrm{mg} / \mathrm{ml})$ and their intestines were collected $2 \mathrm{~h}$ later. In one group, we took samples of only the luminal content from each of the five intestinal segments and analysed the oligosaccharide composition. In the other group, we took the entire tissue for each of the five segments, heat-inactivated potential glycosidases and analysed the glycan composition. For all five intestinal segments, the HPLC oligosaccharide profiles were very similar between luminal aspirates (Fig. 1(A) and Supplementary Fig. S1, available online) and whole tissue lysates (Fig. 1(B) and Supplmentary Fig. S1, available online). These results showed that endogenous intestinal tissue oligosaccharides do not contaminate our HPLC results and that HMO are not degraded during tissue disruption in the sample collection process. Since the collection and handling of samples from the narrow lumen of neonatal intestines were challenging, we chose to use whole tissue lysates in all following experiments.

Next, we assessed whether HMO can be distinguished from other exogenous oligosaccharides present in rat milk or formula. Again, we used 3-d-old rats and separated them into four groups. One group of pups was not fasted and left with the dam until they were killed (DF). All other pups were separated from the dam and fasted for $12 \mathrm{~h}$. One group was immediately killed after the fasting period. The remaining pups were orally administered with either FF or formula that contained HMO at $15 \mathrm{mg} / \mathrm{ml}$ (HMO pulse) and were killed $2 \mathrm{~h}$ later. All five intestinal segments in DF pups showed almost identical oligosaccharide profiles with a single prominent peak (Fig. 1(C)), which was identified as $3^{\prime}$ SL based on HPLC standard retention time and MS analysis. $3^{\prime} \mathrm{SL}$ is the major oligosaccharide in rat milk ${ }^{(30)}$. Accordingly, in fasting 

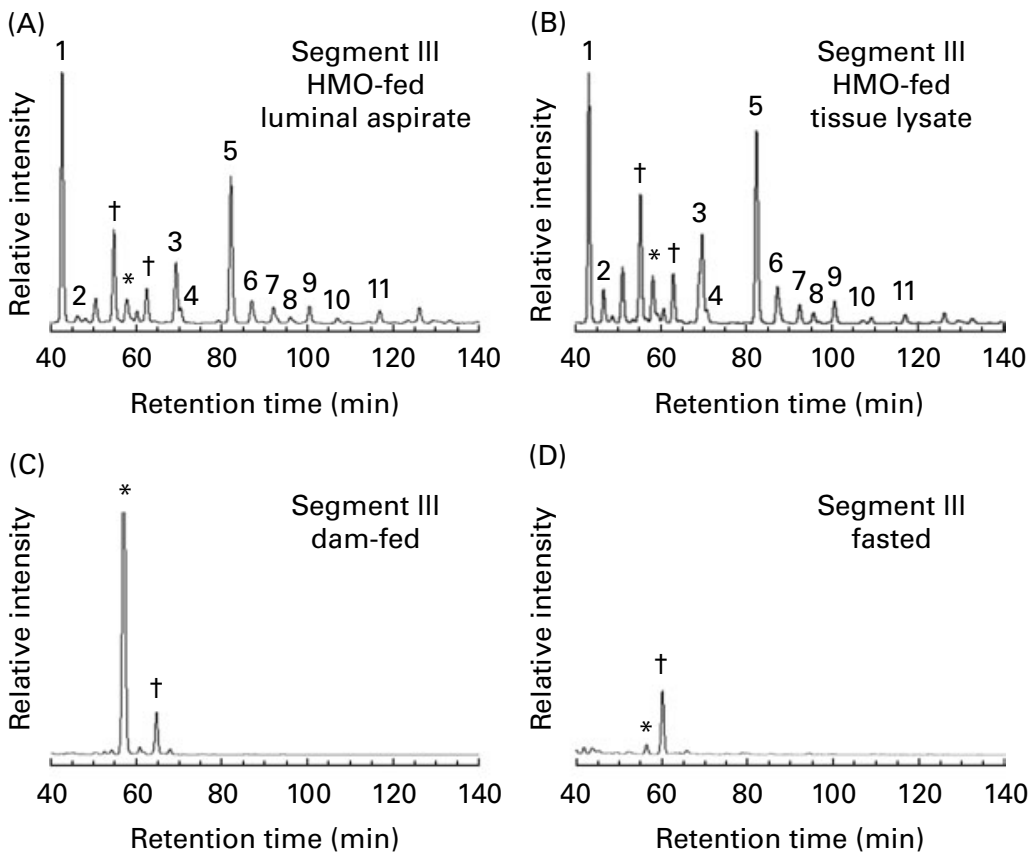

(D)
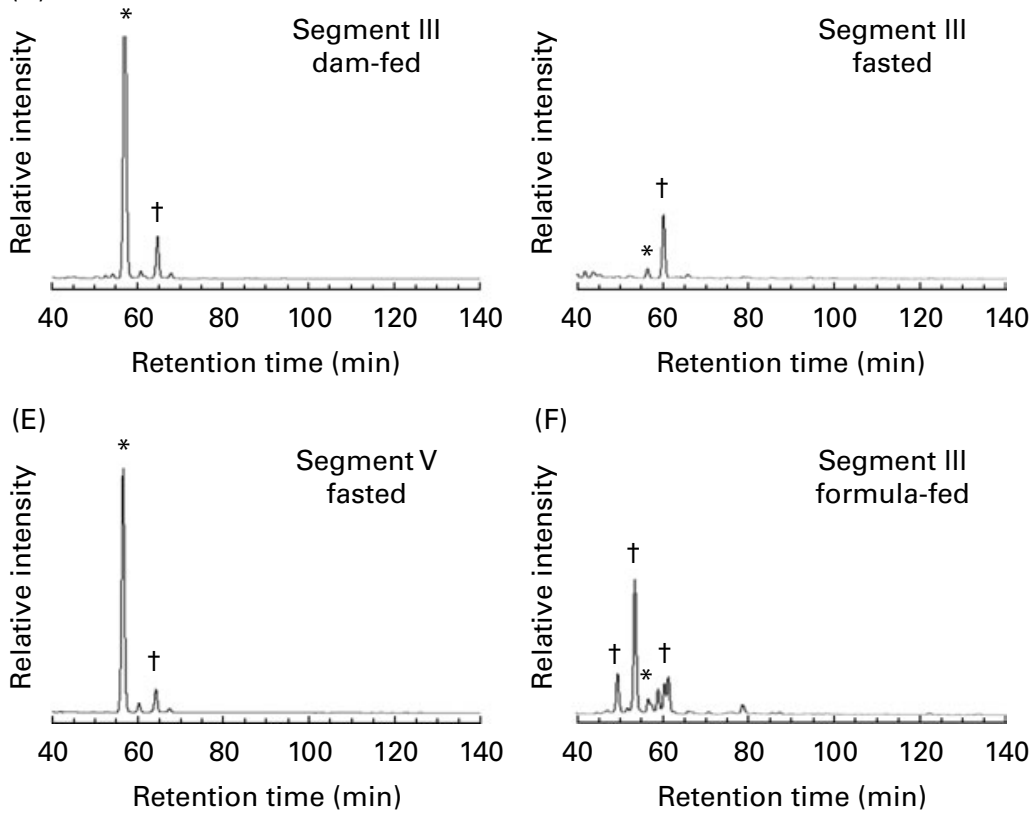

Fig. 1. Human milk oligosaccharides (HMO) can be detected in rat intestines without interference from other dietary or endogenous oligosaccharides and without degradation during sample processing. Representative HPLC chromatograms of oligosaccharides isolated from intestinal segments. Oligosaccharide composition in the third intestinal segment is not different whether (A) luminal aspirates alone or (B) entire tissue lysates are analysed (Supplementary Fig. S1 (available online) shows representative chromatograms from other intestinal sections). (C) $3^{\prime}$-Sialyllactose $\left(^{*}\right)$ is the major oligosaccharide in the intestines of dam-fed pups, (D) almost absent from the first four segments of fasted pups, (E) but still present in the fifth and most distal intestinal segment in fasted pups. (F) HMO are not present in the intestines of pups that received formula alone, without the addition of HMO. Peaks are annotated as follows: (1) 2'-fucosyllactose; (2) 3-fucosyllactose; (3) lacto- $N$-tetraose, (4) lacto- $N$-neotetraose; (5) lacto- $N$-fucopentaose 1; (6) lacto- $N$-fucopentaose 2; (7) lacto- $N$-sialyltetraose b, (8) lacto- $N$-sialyltetraose c; (9) lacto- $N$-difucohexaose; (10) disialyllacto- $N$-tetraose; (11) fucosyllacto- $N$-hexaose. $\dagger$ Unidentified peak.

pups $3^{\prime}$ SL was almost absent from the first four intestinal segments (Fig. 1(D)), but still present in the fifth and most distal segment (Fig. 1(E)). These results show that the presence and composition of HMO along the intestine can be chased without interference by other dietary oligosaccharides as they are cleared from the first four intestinal segments during the $12 \mathrm{~h}$ fasting period prior to an HMO pulse. The fifth and most distal segment may, however, still contain some $3^{\prime}$ SL from pre-fasting milk feeds.

FF contained hardly any oligosaccharides, which corresponds to the very low amount of complex oligosaccharides in bovine milk $^{(31)}$, the basis of the formula we used in the present study. As a consequence, only minor oligosaccharide peaks were detected in the intestinal segments of pups that received FF without the addition of HMO (Fig. 1(F)), indicating that the formula matrix itself does not contaminate the HMO pulse-chase experiments.
Oligosaccharide profiles from the intestinal segments of the pups that received HMO-containing formula (Figs. 1(A) and (B), Fig. 2) were entirely different from pups that were $\mathrm{DF}$, fasted or received formula without HMO. Intestinal oligosaccharide profiles of HMO-fed pups partially resembled the profile of the pooled HMO that were initially added to the orally administered formula. Interestingly, LNFP1 was the main oligosaccharide in the intestinal samples (Fig. 2(D)), but not in the original HMO-containing formula (Fig. 2(A)), suggesting that the HMO composition changes in the intestine.

In an additional set of experiments, we administered one group of pups with formula that contained HMO and then labelled the oligosaccharides with $2 \mathrm{AB}$ after isolation and purification from the intestinal segments, as described earlier (post-labelling). In parallel, another group of pups was administered with formula that contained HMO that were already labelled with $2 \mathrm{AB}$ (pre-labelling). After isolation 


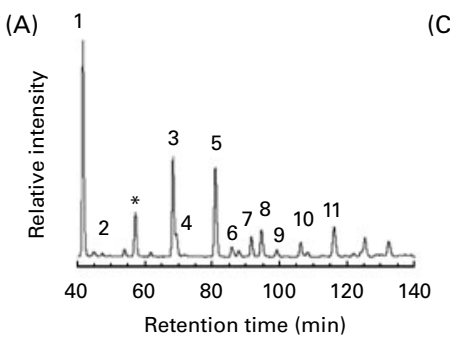

(B)

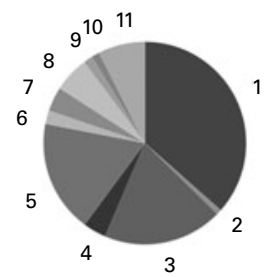

(D)
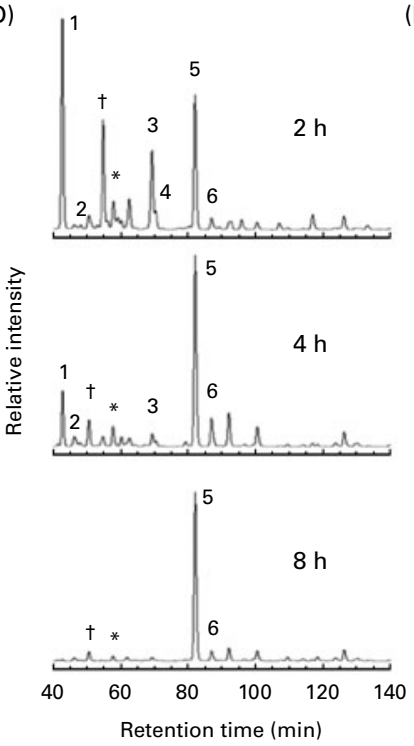

(C)

(1)

2'FL

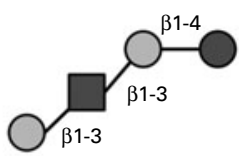

(4) LNnT (type 2 chain)

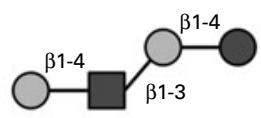

(5)

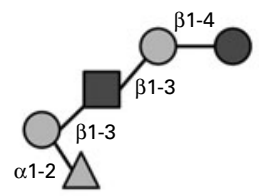

(6)

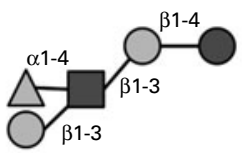

(E)

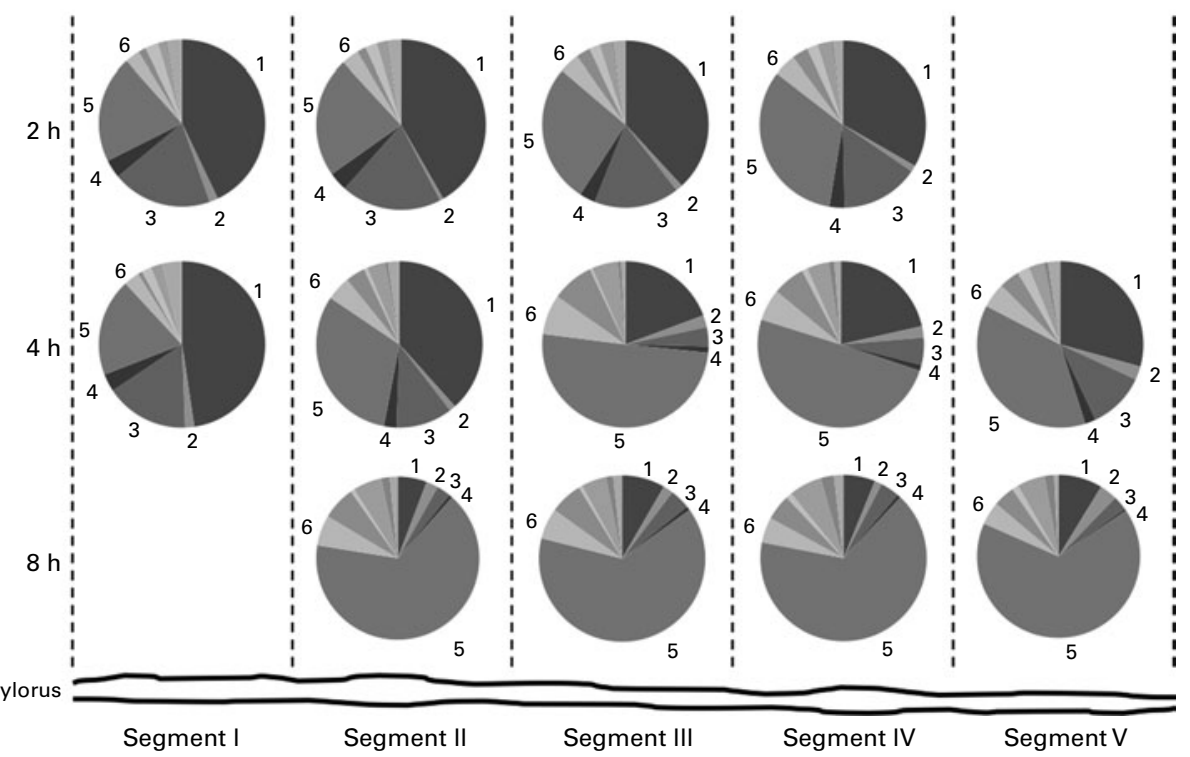

Fig. 2. Human milk oligosaccharides (HMO) composition changes over time and between intestinal segments. HPLC analysis shows that (A) $2^{\prime}$-fucosyllactose $\left(2^{\prime} \mathrm{FL}\right.$, peak 1$)$ is the major oligosaccharide in formula that is supplemented with $\mathrm{HMO}$. The pie chart shows the relative abundance of $2^{\prime} \mathrm{FL}$ and (B) ten other $\mathrm{HMO}$ in the supplemented formula that was orally administered to 3-d-old pups. (C) The structural variety of six of these oligosaccharides is illustrated (O, glucose; O, galactose; $\square, N$-acetyllactosamine; $\Delta$, fucose). (D) Representative HPLC analysis shows that the oligosaccharide composition in the third intestinal segment changes 2, 4 and $8 \mathrm{~h}$ after the rats are orally administered with HMO-containing formula. (E) The relative abundance of eleven oligosaccharides differs between the five different intestinal segments (left to right: proximal to distal) and over time (top to bottom) ( $n 3$ pups for each time point). Peaks are annotated as follows: (1) 2'FL; (2) 3-fucosyllactose (3FL); (3) lacto- $N$-tetraose (LNT), (4) lacto- $N$-neotetraose (LNnT); (5) lacto- $N$-fucopentaose 1 (LNFP1); (6) lacto- $N$-fucopentaose 2 (LNFP2); (7) lacto- $N$-sialyltetraose b, (8) lacto- $N$-sialyltetraose c; (9) lacto- $N$-difucohexaose; (10) disialyllacto- $N$-tetraose; (11) fucosyllacto- $N$-hexaose. ${ }^{*} 3^{\prime}$-Sialyllactose; $†$ unidentified peak.

and purification from the intestinal segments, oligosaccharides were directly subjected to HPLC with fluorescence detection without additional 2AB-labelling. This way, only glycans comprising the reducing end of the administered HMO are detected, omitting possible non-reducing end HMO metabolites and endogenous oligosaccharides. HPLC profiles in the pre-labelled group were almost identical to those in the post-labelled group (Supplementary Figs. S2 and $\mathrm{S} 3$, available online). Again, LNFP 1 was the major oligosaccharide in the intestinal segments, but not in the originally administered HMO-containing formula, confirming our previous results.

\section{Human milk oligosaccharide composition changes over time and between intestinal segments}

Already during method development and validation, we observed that the HMO compositions in the intestinal segments differed from the orally administered HMO. In the following studies, we aimed to identify when and where these changes occur. Following a $12 \mathrm{~h}$ fasting period, we orally administered 3-d-old pups with either FF or formula that contained $\mathrm{HMO}$ at $15 \mathrm{mg} / \mathrm{ml}$ (pulse, Fig. 2(A)-(C)) and then killed the animals 2,4 or $8 \mathrm{~h}$ after the feed (chase). Fig. 2(D) shows how the HMO composition changes over time in the third intestinal segment, which contained some 
Table 1. Relative abundance of selected oligosaccharides in human milk oligosaccharide (HMO)-containing formula and in the five different intestinal segments (I to $\mathrm{V}$, proximal to distal) at 2 , 4 and $8 \mathrm{~h}$ after feeding ( $\mathrm{3}$ rats per time point)

(Mean values with their standard errors)

\begin{tabular}{|c|c|c|c|c|c|c|c|c|c|c|c|c|}
\hline & \multirow{3}{*}{$\begin{array}{c}\mathrm{HMO} \\
\text { in formula }\end{array}$} & \multicolumn{10}{|c|}{ Intestinal segment (proximal to distal) } & \multirow[b]{3}{*}{$\begin{array}{l}\text { One-way } \\
\text { ANOVA }\end{array}$} \\
\hline & & \multicolumn{2}{|c|}{1} & \multicolumn{2}{|c|}{ II } & \multicolumn{2}{|c|}{ III } & \multicolumn{2}{|c|}{ IV } & \multicolumn{2}{|c|}{ v } & \\
\hline & & Mean & SD & Mean & SD & Mean & SD & Mean & SD & Mean & SD & \\
\hline $2^{\prime} \mathrm{FL}$ & $36 \cdot 5$ & & & & & & & & & & & \\
\hline $2 \mathrm{~h}$ & & $42 \cdot 9$ & 1.7 & 41.7 & 2.5 & 38.2 & 3.7 & 33.3 & 8.4 & & & 0.1427 \\
\hline $4 \mathrm{~h}$ & & $47 \cdot 6$ & 1.9 & 38.4 & $13 \cdot 3$ & $19 \cdot 3$ & $11 \cdot 4$ & 21.5 & $7 \cdot 4$ & $29 \cdot 3$ & $6 \cdot 2$ & 0.0157 \\
\hline $8 \mathrm{~h}$ & & & & $5 \cdot 8$ & 4.5 & $8 \cdot 4$ & 6.9 & $6 \cdot 2$ & 4.9 & $8 \cdot 8$ & 7.4 & 0.9011 \\
\hline One-way ANOVA & & & & \multicolumn{2}{|c|}{0.0032} & \multicolumn{2}{|c|}{0.0106} & \multicolumn{2}{|c|}{0.0095} & & & \\
\hline $3 F L$ & 0.8 & & & & & & & & & & & \\
\hline $2 \mathrm{~h}$ & & 1.7 & 0.1 & 0.7 & 0.2 & 1.3 & 0.3 & $1 \cdot 2$ & 0.6 & & & 0.0395 \\
\hline $4 \mathrm{~h}$ & & 1.8 & 0.9 & $1 \cdot 2$ & 0.8 & $2 \cdot 3$ & 1.4 & $2 \cdot 2$ & 0.8 & $2 \cdot 6$ & 1.1 & 0.4856 \\
\hline $8 \mathrm{~h}$ & & & & $2 \cdot 4$ & 1.0 & 1.9 & 0.8 & 1.4 & 0.9 & 3.5 & 0.7 & 0.0869 \\
\hline One-way ANOVA & & & & \multicolumn{2}{|c|}{0.0674} & \multicolumn{2}{|c|}{0.4315} & \multicolumn{2}{|c|}{0.3013} & & & \\
\hline LNT & $19 \cdot 1$ & & & & & & & & & & & \\
\hline $2 \mathrm{~h}$ & & $19 \cdot 4$ & 0.9 & $19 \cdot 2$ & 1.6 & $16 \cdot 6$ & $2 \cdot 6$ & $15 \cdot 2$ & 2.4 & & & 0.0820 \\
\hline $4 \mathrm{~h}$ & & $16 \cdot 2$ & 1.0 & $11 \cdot 0$ & $2 \cdot 7$ & $3 \cdot 7$ & 1.4 & $5 \cdot 3$ & $1 \cdot 2$ & $11 \cdot 1$ & 1.9 & $<0.0001$ \\
\hline $8 \mathrm{~h}$ & & & & $2 \cdot 8$ & 1.4 & 3.9 & $2 \cdot 2$ & 3.7 & $2 \cdot 2$ & $2 \cdot 8$ & 1.4 & 0.8077 \\
\hline One-way ANOVA & & & & \multicolumn{2}{|c|}{0.0002} & \multicolumn{2}{|c|}{0.0004} & \multicolumn{2}{|c|}{0.0008} & & & \\
\hline LNnT & 3.7 & & & & & & & & & & & \\
\hline $2 \mathrm{~h}$ & & $3 \cdot 6$ & 0.1 & 3.7 & 0.4 & $3 \cdot 1$ & 0.2 & 3.0 & 0.5 & & & 0.0873 \\
\hline $4 \mathrm{~h}$ & & 3.5 & 0.6 & 2.5 & 0.3 & $1 \cdot 2$ & 0.4 & 1.2 & 0.1 & $2 \cdot 1$ & 0.8 & 0.0015 \\
\hline $8 \mathrm{~h}$ & & & & 0.7 & 0.5 & 0.8 & 0.4 & 0.7 & 0.4 & 0.5 & 0.3 & 0.6933 \\
\hline One-way ANOVA & & & & \multirow{2}{*}{\multicolumn{2}{|c|}{0.0004}} & \multicolumn{2}{|c|}{0.0002} & \multicolumn{2}{|c|}{0.0007} & & & \\
\hline LNFP1 & $17 \cdot 9$ & & & & & & & & & & & \\
\hline $2 \mathrm{~h}$ & & 20.8 & 0.8 & $22 \cdot 9$ & 1.4 & $27 \cdot 1$ & 3.0 & 32.9 & 9.5 & & & 0.0751 \\
\hline $4 \mathrm{~h}$ & & $19 \cdot 0$ & 5.0 & 31.4 & 11.0 & 50.4 & 7.9 & 49.7 & $4 \cdot 2$ & 37.5 & 5.9 & 0.0011 \\
\hline $8 \mathrm{~h}$ & & & & 65.9 & $8 \cdot 8$ & 63.9 & $10 \cdot 5$ & $66 \cdot 1$ & 5.5 & $66 \cdot 1$ & 6.9 & 0.9834 \\
\hline One-way ANOVA & & & & & & & & & & & & \\
\hline LNFP2 & $2 \cdot 1$ & & & & & & & & & & & \\
\hline $2 \mathrm{~h}$ & & $2 \cdot 8$ & 0.6 & 3.3 & $1 \cdot 1$ & 3.9 & $1 \cdot 1$ & $4 \cdot 3$ & 0.4 & & & 0.2170 \\
\hline $4 \mathrm{~h}$ & & 3.0 & 0.8 & 4.7 & 1.6 & $7 \cdot 8$ & 1.7 & $6 \cdot 2$ & 0.5 & 4.9 & 1.4 & 0.0121 \\
\hline $8 \mathrm{~h}$ & & & & $6 \cdot 0$ & 1.8 & $6 \cdot 0$ & 1.2 & 4.9 & 0.6 & 4.5 & 0.6 & 0.3390 \\
\hline One-way ANOVA & & & & & & & & & & & & \\
\hline LSTb & 3.9 & & & & & & & & & & & \\
\hline $2 \mathrm{~h}$ & & 1.6 & 0.5 & 1.8 & 0.5 & $2 \cdot 1$ & 0.2 & $3 \cdot 2$ & 0.8 & & & 0.0130 \\
\hline $4 \mathrm{~h}$ & & 0.9 & 0.4 & 3.8 & $2 \cdot 2$ & $8 \cdot 2$ & $2 \cdot 1$ & $6 \cdot 3$ & 0.9 & 4.0 & 0.9 & 0.0013 \\
\hline $8 \mathrm{~h}$ & & & & 6.7 & $2 \cdot 1$ & $6 \cdot 4$ & 0.7 & $5 \cdot 0$ & 0.2 & $4 \cdot 3$ & 0.2 & 0.0906 \\
\hline One-way ANOVA & & & & & & & & & & & & \\
\hline LSTC & $5 \cdot 7$ & & & & & & & & & & & \\
\hline $2 \mathrm{~h}$ & & 2.5 & 0.7 & $2 \cdot 2$ & 0.3 & $2 \cdot 0$ & 0.4 & $2 \cdot 1$ & 0.2 & & & 0.5175 \\
\hline $4 \mathrm{~h}$ & & 1.9 & 0.5 & 0.7 & 0.7 & 0.5 & 0.3 & 1.0 & 0.3 & $2 \cdot 3$ & 1.3 & 0.0467 \\
\hline $8 \mathrm{~h}$ & & & & 0.8 & 0.2 & 0.7 & 0.6 & $1 \cdot 1$ & 0.4 & 1.3 & 0.8 & 0.4582 \\
\hline One-way ANOVA & & & & & & & & & & & & \\
\hline LNDFH & 1.5 & & & & & & & & & & & \\
\hline $2 \mathrm{~h}$ & & 1.4 & 0.3 & 1.7 & 0.9 & $2 \cdot 4$ & 1.0 & $2 \cdot 8$ & 0.6 & & & 0.1867 \\
\hline $4 \mathrm{~h}$ & & 1.8 & 0.6 & 3.6 & 1.5 & $5 \cdot 0$ & $1 \cdot 8$ & $4 \cdot 3$ & 1.4 & 3.0 & 0.4 & 0.0762 \\
\hline
\end{tabular}


of the mid and distal jejunum, but not the colon. Subtle changes were apparent as early as $2 \mathrm{~h}$ after the feeding. After $8 \mathrm{~h}$, low-molecular HMO like $2^{\prime} \mathrm{FL}$ or lacto- $N$-tetraose were almost gone, while higher-molecular HMO, like LNFP1, became the most dominant HMO. To rule out that retention times of unknown metabolites coincided with those of HMO standards, the identity of all major peaks was confirmed by MS.

We calculated the relative abundance of all major HMO in the different intestinal segments and at different time points (Table 1 and Fig. 2(E)), but excluded $3^{\prime}$ SL as some of it was carried over from pre-fasting milk feeds. As expected, at $2 \mathrm{~h}$ after feeding, oligosaccharide profiles of the most distal intestinal sections did not resemble HMO, but rat milk with its characteristic 3'SL peak, and the data were omitted from the analysis. Most intriguingly and against previous beliefs, changes in the oligosaccharide profiles already occurred in the third intestinal segment, which represents mostly the distal jejunum, but not the colon. The relative abundance of $2^{\prime} \mathrm{FL}$ decreased from the orally administered 36.5 to $19 \cdot 3$ (SD 11.4 ) and $8.4(\mathrm{SD} 6.9) \%$ after 4 and $8 \mathrm{~h}$, respectively. Lacto$N$-tetraose dropped from the original $19 \cdot 1$ to 3.7 (SD 1.4) \% at $4 \mathrm{~h}$ and to 3.9 (SD $2 \cdot 2$ ) \% at $8 \mathrm{~h}$. Its structural isomer lacto$N$-neotetraose dropped from 3.7 to 1.2 (SD 0.4 ) \% at $4 \mathrm{~h}$ and to $0.8(\mathrm{sD} 0.4) \%$ at $8 \mathrm{~h}$. In contrast, the relative abundance of the fucosylated HMO LNFP1 increased from 17.9 to $50 \cdot 4$ (SD 7.9$) \%$ at $4 \mathrm{~h}$ and to 63.9 (SD 10.5) $\%$ at $8 \mathrm{~h}$. Its isomer LNFP2 also increased over time. The relative abundance of the sialylated HMO lacto- $N$-sialyltetraose $\mathrm{b}$ increased from $3.9 \%$ in the HMO-containing formula to 8.2 (SD 2.1 ) $\%$ at $4 \mathrm{~h}$ and to 6.4 (SD 0.7$) \%$ at $8 \mathrm{~h}$. Its isomer lacto- $N$-sialyltetraose c, however, dropped from $5.7 \%$ in the formula to 0.5 (SD 0.3 ) \% at $4 \mathrm{~h}$ and to 0.7 (sD 0.6 ) \% at $8 \mathrm{~h}$. We did not observe any significant changes in the relative abundance of disialyllacto$N$-tetraose or fucosyllacto- $N$-hexaose between different intestinal segments or within the same segment at different time points.

It is noteworthy to mention that some of the compositional changes occurred already in the more proximal second segment. The relative abundance of $2^{\prime} \mathrm{FL}$ decreased from the initial 36.5 to 5.8 (SD 4.5 ) \% at $8 \mathrm{~h}$. Lacto- $N$-tetraose decreased from $19 \cdot 1$ to $2 \cdot 8$ (SD 1.4 ) $\%$ and lacto- $N$-neotetraose dropped from 3.7 to 0.7 (SD 0.5$) \%$. At the same time, LNFP1 increased from 17.9 to 65.9 (SD 8.8)\%. While lacto- $N$-sialyltetraose b increased from 3.9 to 6.7 (SD $2 \cdot 1$ ) \%, its structural isomer lacto- $N$-sialyltetraose c decreased from 5.7 to 0.8 (SD 0.2 ) \% in the very same second segment and within the same time frame.

\section{Rat serum and urine contains 3'-sialyllactose, but hardly any other human milk oligosaccharides}

Studies on human infants have shown that HMO are partially absorbed and excreted with the urine ${ }^{(25,26)}$. Thus, we examined whether the same applies for rat pups. Indeed, we detected a prominent $3^{\prime} \mathrm{SL}$ peak in both the serum and urine of DF pups (Fig. 3(A) and (B)). In pups that had fasted for $12 \mathrm{~h}, 3^{\prime} \mathrm{SL}$ was almost absent from the serum (Fig. 3(C)), but still present in the urine (Fig. 3(D)), although at lower concentrations than in the DF pups. When we administered the fasted 
pups with HMO-containing formula, $3^{\prime} \mathrm{SL}$ appeared in $1 \mathrm{~h}$ post-feeding serum, reached maximum levels between 4 and $6 \mathrm{~h}$ and was still detectable after $8 \mathrm{~h}$ (Fig. 3(E)). $3^{\prime} \mathrm{SL}$ also appeared in the $8 \mathrm{~h}$ urine sample (Fig. 3(F)).

While $3^{\prime}$ SL was not the most abundant oligosaccharide in the HMO-containing formula (Fig. 2(A)), the serum and urine of HMO-fed pups almost exclusively contained 3'SL (Fig. 3(E) and (F)). We used MS (data not shown) as well as HPLC following neuraminidase digestion (Fig. $3(\mathrm{G})$ ) and confirmed that the observed peak indeed represented $3^{\prime} \mathrm{SL}$. Minor peaks appeared in the urine of HMO-fed pups (Fig. 3(F)) that were absent from the urine of pups that were DF, fasted or received formula without HMO. Standard retention times suggested that these peaks represented $2^{\prime} \mathrm{FL}$ and LNFP1, which we confirmed by MS as well as HPLC after fucosidase digestion (Fig. 3(H)). The dominance of $3^{\prime} \mathrm{SL}$ over other HMO in the serum and the urine of HMO-fed pups indicates an absorption mechanism that is specific for $3^{\prime} \mathrm{SL}$.
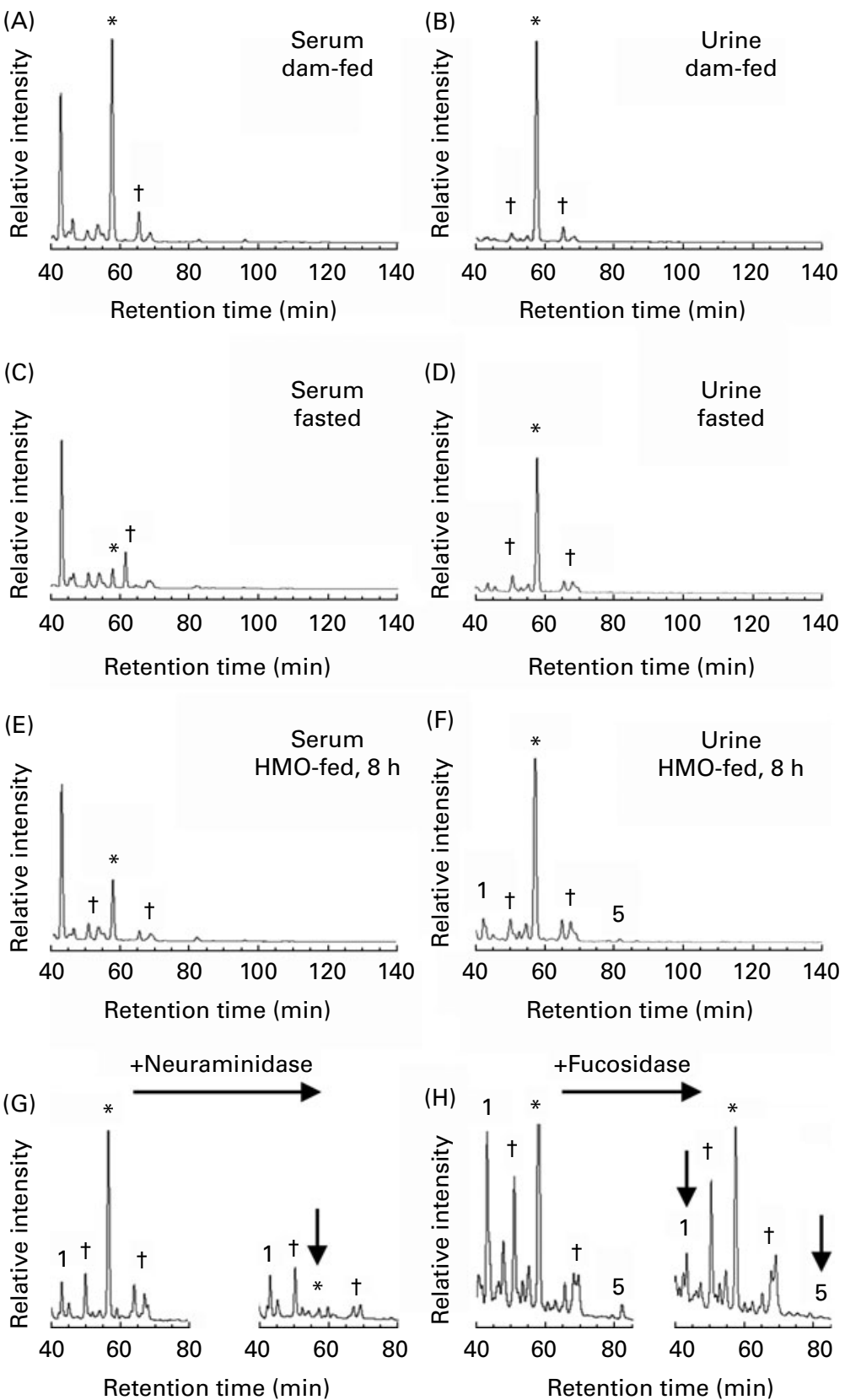

Fig. 3. Rat serum and urine contains $3^{\prime}$-sialyllactose $\left(3^{\prime} \mathrm{SL}\right)$, but hardly any other human milk oligosaccharides (HMO). Representative HPLC chromatograms show that $3^{\prime} S L\left(^{*}\right)$ appears in the (A) serum and (B) urine of dam-fed rats. (C) $3^{\prime} \mathrm{SL}$ disappears from the rat serum after a $12 \mathrm{~h}$ fasting period, (D) but is still present in urine. (E) Serum and (F) urine that were collected of rats $8 \mathrm{~h}$ after they received HMO-containing formula also contained $3^{\prime} \mathrm{SL}$, but hardly any other oligosaccharides. The urine samples contained small amounts of $2^{\prime}$-fucosyllactose (2'FL, peak 1 ) and lacto- $N$-fucopentaose 1 (LNFP1, peak 5 ). (G) The presence of $3^{\prime}$ SL in urine was confirmed by neuraminidase digestion. The $3^{\prime} \mathrm{SL}$ peak in the mock-treated sample (left) disappeared in the neuraminidase-treated sample (right). (H) The presence of $2^{\prime} \mathrm{FL}$ and LNFP1 in urine was confirmed by fucosidase digestion. The 2'FL and LNFP1 peaks in the mock-treated sample (left) disappeared after fucosidase treatment (right). †Unidentified peak. 


\section{Galacto-oligosaccharides are selectively digested in the} small intestine and do not appear in the urine

GOS are added to some infant formula to mimic some of the benefits of $\mathrm{HMO}$ and, like $\mathrm{HMO}$, are considered to be indigestible in the small intestine. When we administered fasted pups with GOS-containing formula at $8 \mathrm{mg} / \mathrm{ml}$ (Fig. 4(A)), GOS composition changed within the first $2 \mathrm{~h}$ and already in the third intestinal segment (Fig. 4(B)). After $4 \mathrm{~h}$, different diand trisaccharide peaks disappeared from the HPLC profile (Fig. 4(C)). After $8 \mathrm{~h}$, disaccharides were almost absent and some of the tri- and tetrasaccharides had also disappeared, while others were still present (Fig. 4(D)). These results indicate a selective degradation of certain GOS while others remain intact.

Oligosaccharide profiles in 6 and $8 \mathrm{~h}$ urine from GOS-fed rats contained a dominant $3^{\prime}$ SL peak (Fig. 4(E)) and closely resembled urine samples from pups that received $\mathrm{FF}$ without GOS (Fig. 4(F)). These results indicate that GOS are not excreted with the urine.

\section{Discussion}

HMO and GOS were previously considered to be indigestible in the small intestine and to reach the colon and other target organs in the same composition as originally secreted with the mother's milk. The present results now show that this assumption may be incorrect - at least for neonatal rats.

Changes in the composition of HMO occur as early as $2 \mathrm{~h}$ post-feeding and already within the second and third intestinal segment (Fig. 2 and Table 1). Due to the small size of 3-d-old rat pups, we were unable to separate their intestines by anatomical distinction as duodenum, jejunum, ileum or colon. Instead, we separated the intestines in five equal-length segments, with the second and third segments mostly spanning the jejunum, but not the colon. We therefore conclude that the composition of HMO and GOS already changes before they reach the colon

Studies in tissue culture models suggest that HMO are antiadhesive antimicrobials that serve as soluble decoy receptors and prevent the attachment of potential pathogens to the
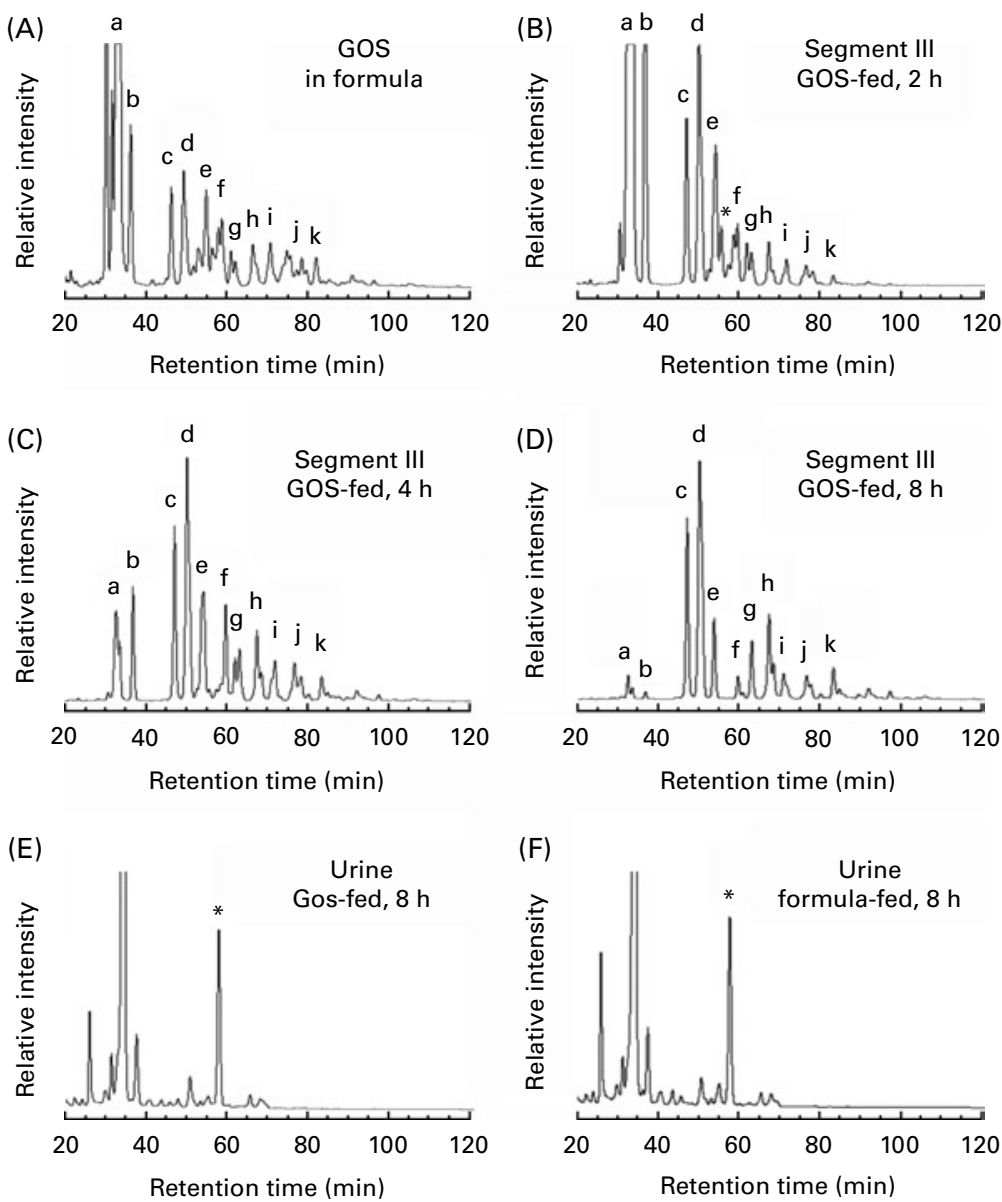

Fig. 4. Galacto-oligosaccharides (GOS) are selectively digested in the small intestine and do not appear in the urine. HPLC chromatograms of oligosaccharides in formula that was supplemented with (A) GOS and in the third intestinal segment (B) $2 \mathrm{~h},(\mathrm{C}) 4 \mathrm{~h}$ and (D) $8 \mathrm{~h}$ after rats were administered with the GOS-containing formula. HPLC chromatograms of oligosaccharides in the urine of pups that received either (E) GOS-supplemented formula or (F) formula alone. GOS peaks are marked with small letters for easier comparison between the different chromatograms, but exact structural composition represented by each peak is not known. GOS disaccharides elute before $40 \mathrm{~min}$, trisaccharides elute between 40 and $60 \mathrm{~min}$, tetrasaccharides elute between 60 and 80 min and oligosaccharides with five or more monomers elute after $80 \mathrm{~min}$. ${ }^{*} 3^{\prime}$-Sialyllactose. 
infant's intestinal epithelial surfaces, which is often an essential step for pathogens to cause disease ${ }^{(3)}$. In addition, HMO modulate proliferation, differentiation and apoptosis, as well as surface glycosylation in intestinal epithelial cells ${ }^{(5,6,32)}$. These in vitro studies apply HMO to the cultured intestinal epithelial cells in the same composition as originally secreted with the mother's milk. The present results suggest that some of the in vitro-active HMO may, in fact, never reach the intestinal epithelium in the distal small intestine and colon in vivo.

The compositional changes are structure-specific as the relative abundance of the $\alpha 1$-2-fucosylated trisaccharides $2^{\prime}$ FL decreases, but that of the $\alpha 1$-2-fucosylated pentasaccharide LNFP1 increases (Fig. 2 and Table 1). The same is true for sialylated HMO as the relative abundance of lacto- $N$ sialyltetraose b significantly increases and that of its isomer lacto- $N$-sialyltetraose c significantly decreases throughout the intestinal passage and over time. While we cannot annotate each individual GOS isomer in the HPLC chromatograms, it is clear that some GOS structures disappear and others resist degradation (Fig. 4). GOS are a heterogeneous group of oligosaccharides with different glycosidic linkages between the Gal monomers, and it is likely that some linkages can be cleaved during the intestinal passage while others resist. Due to the lack of an inert internal oligosaccharide reference standard, we cannot provide absolute HMO or GOS concentrations for each of the intestinal segments and time points, but it is evident from the present results that HMO and GOS undergo dramatic compositional changes. It remains, however, unknown whether these changes are caused by endogenous intestinal enzymes or by microbial enzymes. Studies in gnotobiotic animals may be able to answer this question.

The major challenge of the present study lies in the rat model itself. Differences in intestine anatomy, physiology and biochemistry between rats and human subjects make it difficult to predict whether the present results in rat pups translate to human neonates ${ }^{(33)}$. The most important difference in this particular context may be the microbial colonisation in proximal parts of the gastrointestinal tract. The amount of bacteria in the proximal small intestine and even in the stomach is several orders of magnitudes higher in rats than it is in human subjects ${ }^{(33)}$. In addition, the composition of intestinal bacterial communities is probably going to differ between laboratory rats and human neonates, which at least partially determines when, where and to what extent dietary oligosaccharides are metabolised.

The present results raise two fundamental questions: (1) are rats and other laboratory animals like mice or piglets suitable models to study the effects of HMO? and (2) are HMO indigestible in the small intestine of human neonates and do they reach the colon in the same composition as in mother's milk as originally assumed or do the present results translate - at least in part - from rats to human subjects and the HMO composition changes before reaching the colon? HMO resist a low $\mathrm{pH}$ as well as degradation through enzymes from the pancreas and brush-border membranes ${ }^{(15,16)}$, resembling some of the conditions in the infant's stomach and small intestine. The data, however, stem from in vitro incubation studies. There are currently no in vivo data on HMO composition in the small intestine of human infants. At the same time, recent studies show that the oligosaccharide composition in the faeces of breast-fed infants is different from that in the mother's milk ${ }^{(21-23)}$. It remains unknown when, where and how these changes occur between the time of ingestion and the time of excretion.

Another striking observation in the present study is the selective absorption and urinary excretion of $3^{\prime}$ SL. Human milk contains a variety of different oligosaccharides and so does the urine of breast-fed infants ${ }^{(25,26,34)}$. Rat milk, however, contains mostly $3^{\prime} \mathrm{SL}^{(30)}$ and it appears that rat pups selectively absorb 3'SL even if high amounts of other oligosaccharides (HMO or GOS) are administered with the diet (Fig. 3). These results speak against the use of rats in studies that aim to investigate the effects of HMO in extra-intestinal target organs and tissues. Whether HMO absorption and urinary excretion in other animal models, like mice or piglets, more closely resemble those in human neonates needs to be further investigated.

\section{Supplementary material}

To view supplementary material for this article, please visit http://dx.doi.org/10.1017/S0007114512005727

\section{Acknowledgements}

We are grateful to Caroline Nissan for her technical assistance with HMO purification and analysis. This project has been funded in part by the National Institutes of Health grant K99/R00 DK078668 (L. B.). E. J.-K., C. M. and L. B. have no conflicts of interest to declare. Authors' contributions were as follows: L. B. and E. J.-K. designed research; E. J.-K. and C. M. conducted research; E. J.-K and L. B. analysed data; E. J.-K and L. B. wrote the paper. L. B. had the primary responsibility for the final content. All authors read and approved the final manuscript.

\section{References}

1. Bode L (2006) Recent advances on structure, metabolism, and function of human milk oligosaccharides. J Nutr $\mathbf{1 3 6}$ 2127-2130.

2. Bode L (2009) Human milk oligosaccharides: prebiotics and beyond. Nutr Rev 67, Suppl. 2, S183-S191.

3. Kunz C, Rudloff S, Baier W, et al. (2000) Oligosaccharides in human milk: structural, functional, and metabolic aspects. Annu Rev Nutr 20, 699-722.

4. Newburg DS, Ruiz-Palacios GM \& Morrow AL (2005) Human milk glycans protect infants against enteric pathogens. Annu Rev Nutr 25, 37-58.

5. Kuntz S, Kunz C \& Rudloff S (2009) Oligosaccharides from human milk induce growth arrest via G2/M by influencing growth-related cell cycle genes in intestinal epithelial cells. Br J Nutr 101, 1306-1315.

6. Kuntz S, Rudloff S \& Kunz C (2008) Oligosaccharides from human milk influence growth-related characteristics of intestinally transformed and non-transformed intestinal cells. Br J Nutr 99, 462-471. 
7. Wang B (2009) Sialic acid is an essential nutrient for brain development and cognition. Annu Rev Nutr 29, 177-222.

8. Jantscher-Krenn E, Zherebtsov M, Nissan C, et al. (2012) The human milk oligosaccharide disialyllacto- $N$-tetraose prevents necrotising enterocolitis in neonatal rats. Gut 61, 1417-1425.

9. Kobata A (2010) Structures and application of oligosaccharides in human milk. Proc Jpn Acad Ser B Phys Biol Sci 86, $731-747$

10. Bode L, Kunz C, Muhly-Reinholz M, et al. (2004) Inhibition of monocyte, lymphocyte, and neutrophil adhesion to endothelial cells by human milk oligosaccharides. Thromb Haemost 92, 1402-1410.

11. Bode L, Rudloff S, Kunz C, et al. (2004) Human milk oligosaccharides reduce platelet-neutrophil complex formation leading to a decrease in neutrophil beta 2 integrin expression. J Leukoc Biol 76, 820-826.

12. Jantscher-Krenn E, Lauwaet T, Bliss LA, et al. (2012) Human milk oligosaccharides reduce Entamoeba histolytica attachment and cytotoxicity in vitro. Br J Nutr 108, 1839-1846.

13. Morrow AL, Ruiz-Palacios GM, Altaye M, et al. (2004) Human milk oligosaccharides are associated with protection against diarrhea in breast-fed infants. J Pediatr 145, 297-303.

14. Ruiz-Palacios GM, Cervantes LE, Ramos P, et al. (2003) Campylobacter jejuni binds intestinal $\mathrm{H}(\mathrm{O})$ antigen (Fuc alpha 1, $2 \mathrm{Gal}$ beta 1, 4GlcNAc), and fucosyloligosaccharides of human milk inhibit its binding and infection. $J$ Biol Chem 278, 14112-14120.

15. Engfer MB, Stahl B, Finke B, et al. (2000) Human milk oligosaccharides are resistant to enzymatic hydrolysis in the upper gastrointestinal tract. Am J Clin Nutr 71, 1589-1596.

16. Gnoth MJ, Kunz C, Kinne-Saffran E, et al. (2000) Human milk oligosaccharides are minimally digested in vitro. J Nutr $\mathbf{1 3 0}$, 3014-3020.

17. Asakuma S, Hatakeyama E, Urashima T, et al. (2011) Physiology of consumption of human milk oligosaccharides by infant gut-associated bifidobacteria. J Biol Chem 286, 34583-34592.

18. LoCascio RG, Ninonuevo MR, Freeman SL, et al. (2007) Glycoprofiling of bifidobacterial consumption of human milk oligosaccharides demonstrates strain specific, preferential consumption of small chain glycans secreted in early human lactation. J Agric Food Chem 55, 8914-8919.

19. Marcobal A, Barboza M, Froehlich JW, et al. (2010) Consumption of human milk oligosaccharides by gut-related microbes. J Agric Food Chem 58, 5334-5340.

20. Marcobal A, Barboza M, Sonnenburg ED, et al. (2011) Bacteroides in the infant gut consume milk oligosaccharides via mucus-utilization pathways. Cell Host Microbe $\mathbf{1 0}$, 507-514.
21. Albrecht S, Schols HA, van den Heuvel EG, et al. (2010) CE-LIF-MS $\mathrm{n}$ profiling of oligosaccharides in human milk and feces of breast-fed babies. Electrophoresis 31, $1264-1273$

22. Albrecht S, Schols HA, van den Heuvel EG, et al. (2011) Occurrence of oligosaccharides in feces of breast-fed babies in their first six months of life and the corresponding breast milk. Carbohydr Res 346, 2540-2550.

23. Albrecht S, Schols HA, van Zoeren D, et al. (2011) Oligosaccharides in feces of breast- and formula-fed babies. Carbobydr Res 346, 2173-2181.

24. Gnoth MJ, Rudloff S, Kunz C, et al. (2001) Investigations of the in vitro transport of human milk oligosaccharides by a Caco-2 monolayer using a novel high performance liquid chromatography-mass spectrometry technique. $J$ Biol Chem 276, 34363-34370.

25. Rudloff S, Obermeier S, Borsch C, et al. (2006) Incorporation of orally applied (13)C-galactose into milk lactose and oligosaccharides. Glycobiology 16, 477-487.

26. Rudloff S, Pohlentz G, Borsch C, et al. (2012) Urinary excretion of in vivo ${ }^{13} \mathrm{C}$-labelled milk oligosaccharides in breastfed infants. BrJ Nutr 107, 957-963.

27. Fuhrer A, Sprenger N, Kurakevich E, et al. (2010) Milk sialyllactose influences colitis in mice through selective intestinal bacterial colonization. J Exp Med 207, 2843-2854.

28. German JB, Freeman SL, Lebrilla CB, et al. (2008) Human milk oligosaccharides: evolution, structures and bioselectivity as substrates for intestinal bacteria. Nestle Nutr Workshop Ser Pediatr Program 62, 205-218, discussion 218-222.

29. Barlow B \& Santulli TV (1975) Importance of multiple episodes of hypoxia or cold stress on the development of enterocolitis in an animal model. Surgery 77, 687-690.

30. Urashima T, Saito T, Nakamura T, et al. (2001) Oligosaccharides of milk and colostrum in non-human mammals. Glycoconj J 18, 357-371.

31. Tao N, DePeters EJ, Freeman S, et al. (2008) Bovine milk glycome. J Dairy Sci 91, 3768-3778.

32. Angeloni S, Ridet JL, Kusy N, et al. (2005) Glycoprofiling with micro-arrays of glycoconjugates and lectins. Glycobiology 15, 31-41.

33. Kararli TT (1995) Comparison of the gastrointestinal anatomy, physiology, and biochemistry of humans and commonly used laboratory animals. Biopharm Drug Dispos 16, 351-380.

34. Rudloff S, Pohlentz G, Diekmann L, et al. (1996) Urinary excretion of lactose and oligosaccharides in preterm infants fed human milk or infant formula. Acta Paediatr 85, 598-603. 